\title{
Concomitant Medication Use Duration
}

National Cancer Institute

\section{Source}

National Cancer Institute. Concomitant Medication Use Duration. NCI Thesaurus. Code C83224.

The period of time from start to finish of concomitant medication usage. 